\title{
GENERALIZED HYPERGROUPS AND ORTHOGONAL POLYNOMIALS
}

\section{RUPERT LASSER, JOSEF OBERMAIER ${ }^{\text {}}$ and HOLGER RAUHUT}

(Received 4 July 2005; revised 8 March 2006)

Communicated by G. Willis

\begin{abstract}
The concept of semi-bounded generalized hypergroups (SBG hypergroups) is developed. These hypergroups are more special than generalized hypergroups introduced by Obata and Wildberger and more general than discrete hypergroups or even discrete signed hypergroups. The convolution of measures and functions is studied. In the case of commutativity we define the dual objects and prove some basic theorems of Fourier analysis. Furthermore, we investigate the relationship between orthogonal polynomials and generalized hypergroups. We discuss the Jacobi polynomials as an example.
\end{abstract}

2000 Mathematics subject classification: primary 43A62, 43A99, 46J10, 05E35, 33C80.

Keywords and phrases: generalized hypergroup, semi-bounded generalized hypergroup, bounded generalized hypergroups, signed hypergroup, discrete hypergroup. convolution, dual object, Fourier transform, orthogonal polynomials, Jacobi polynomials.

\section{Introduction}

Locally compact hypergroups were independently introduced around the 1970's by Dunkl [4], Jewett [7] and Spector [14]. They generalized the concepts of locally compact groups with the purpose of doing standard harmonic analysis. Similar structures had been studied earlier, in the 1950s, by Berezansky and colleagues, and even earlier in works of Delsarte and Levitan.

Later on, results of harmonic analysis on hypergroups were transferred to different applications. For example, a Bochner theorem is used essentially in the context of weakly stationary processes indexed by hypergroups, see [9] and [11]. Hypergroup structure is also heavily used in probability theory, see the monograph [2], and in approximation with respect to orthogonal polynomial sequences, see [5] and [10].

(C) 2007 Australian Mathematical Society 1446-7887/07 \$A2.00+0.00 
However, the whole set of axioms (see [2]) is not used in these application areas. Concentrating on orthogonal polynomials, Obata and Wildberger [12] studied a very general concept called 'generalized hypergroups'. The purpose of the present paper is to derive results of harmonic analysis for generalized hypergroups in more detail. Our main interest is to include all orthogonal polynomial systems with respect to a compactly supported orthogonalization measure in our investigations.

\section{Semi-bounded generalized hypergroups}

The discrete structure of a generalized hypergroup was introduced by Obata and Wildberger in [12]. Let us recall the basic definitions.

DEFINITION 2.1. A generalized hypergroup is a pair $\left(\mathcal{K}, \mathcal{A}_{0}\right)$, where $\mathcal{A}_{0}$ is a *-algebra over $\mathbb{C}$ with unit $c_{0}$ and $\mathcal{K}=\left\{c_{k}, k \in K\right\}$ is a countable subset of $\mathcal{A}_{0}$ containing $c_{0}$ that satisfies the following axioms.

(A1) $\mathcal{K}^{*}=\mathcal{K}$.

(A2) $\mathcal{K}$ is a linear basis of $\mathcal{A}_{0}$, that is, every $a \in \mathcal{A}_{0}$ admits a unique expression of the form $a=\sum_{n} \alpha_{n} c_{n}$ with only finitely many nonzero $\alpha_{i} \in \mathbb{C}$.

(A3) The structure constants or linearization coefficients $g(n, m, k) \in \mathbb{C}$, which are defined by $c_{n} c_{m}=\sum_{k} g(n, m, k) c_{k}$, satisfy the condition

$$
g(n, m, 0) \begin{cases}>0 & \text { if } c_{n}^{*}=c_{m}, \\ =0 & \text { if } c_{n}^{*} \neq c_{m} .\end{cases}
$$

A generalized hypergroup is called hermitian if $c_{n}^{*}=c_{n}$, commutative if $c_{n} c_{m}=c_{m} c_{n}$, real if $g(n, m, k) \in \mathbb{R}$, positive if $g(n, m, k) \geq 0$, and normalized if $\sum_{j} g(n, m, j)=1$ for all $n, m, k$.

A bijection on $K$ is defined by

$$
c_{\tilde{n}}=c_{n}^{*} .
$$

Further, let

$$
h(n)=g(\tilde{n}, n, 0)^{-1}
$$

Due to (A3) we have $h(n)>0$ for all $n$ and $h(0)=1$. If $\mathcal{K}$ is hermitian or commutative, then $h(n)=h(\tilde{n})$. In the following lemma some useful properties of the structure constants are summarized. 
LEMMA 2.2. The structure constants fulfill the following equalities:

$$
\begin{aligned}
g(n, 0, k) & =g(0, n, k)=\delta_{n k}, \\
g(n, m, k) & =\frac{g(\tilde{m}, \tilde{n}, \tilde{k}),}{h(m) g(n, m, k)}=h(k) g(\tilde{k}, n, \tilde{m}), \quad \text { and } \\
\sum_{k} g(n, m, k) g(k, l, j) & =\sum_{k} g(n, k, j) g(m, l, k) \quad \text { for all } n, m, l, j .
\end{aligned}
$$

PROOF. For (2.3)-(2.5) see [12, Lemma 1.1]. Now, on the one hand we have $\left(c_{n} c_{m}\right) c_{l}=\sum_{k, j} g(n, m, k) g(k, l, j) c_{j}$ and on the other hand we have $c_{n}\left(c_{m} c_{l}\right)=$ $\sum_{k, j} g(m, l, k) g(n, k, j) c_{j}$. From the associativity of $\mathcal{A}_{0}$ and the linear independence of the set $\mathcal{K}$, (2.6) follows.

We define translation operators $L_{n}, \overline{L_{n}}$ for complex valued functions $f$ on $K$ by

$$
L_{n} f(m)=\sum_{k} g(n, m, k) f(k) \quad \text { and } \quad \overline{L_{n}} f(m)=\sum_{k} \overline{g(n, m, k)} f(k) .
$$

Given $f$, the function $\tilde{f}$ is defined by $\tilde{f}(n)=f(\tilde{n})$.

LEMMA 2.3. For $f, g$ with finite support and all $n \in K$,

$$
\sum_{m} L_{n} f(m) g(m) h(m)=\sum_{m} f(m) L_{\tilde{m}} \tilde{g}(n) h(m)=\sum_{m} f(m)\left(\overline{L_{\tilde{n}}} g\right)(m) h(m) .
$$

PROOF. We use (2.4) and (2.5) to obtain

$$
\begin{aligned}
\sum_{m}\left(L_{n} f\right)(m) g(m) h(m) & =\sum_{m, k} g(\tilde{k}, n, \tilde{m}) h(k) f(k) g(m) \\
& =\sum_{k} f(k) L_{\tilde{k}} \tilde{g}(n) h(k) \\
& =\sum_{k \cdot m} f(k) \overline{g(\tilde{n}, k, m)} g(m) h(k) \\
& =\sum_{k} f(k) \overline{\left(\overline{L_{\tilde{n}}} g\right)(k) h(k) .}
\end{aligned}
$$

We write $v(k)=v(\{k\})$ for a discrete measure $\nu$ on $K$. Let $\epsilon_{n}$ denote the Diracmeasure at $n \in K$, that is, $\epsilon_{n}(k)=1$ if $k=n$ and $\epsilon_{n}(k)=0$ otherwise.

Definition 2.4. A positive discrete measure $\omega \neq 0$ on $K$ is called (left) Haar measure if, for all $f$ with finite support and all $n \in K$,

$$
\sum_{m} L_{n} f(m) \omega(m)=\sum_{m} f(m) \omega(m) .
$$


THEOREM 2.5. A Haar measure exists if and only if $\mathcal{K}$ is normalized. In that case all Haar measures $\omega$ are determined by $\omega=\alpha h, \alpha>0$.

PROOF. Let us assume that there exists a Haar measure $\omega$. Due to (A3) we get

$$
\begin{aligned}
h(n)^{-1} \omega(n) & =\sum_{m} g(\tilde{n}, m, 0) \omega(m)=\sum_{m} L_{\tilde{n}} \epsilon_{0}(m) \omega(m) \\
& =\sum_{m} \epsilon_{0}(m) \omega(m)=\omega(0),
\end{aligned}
$$

which yields $\omega(n)=\omega(0) h(n)$. Now let $\omega=\alpha h$. It suffices to consider $f=\epsilon_{k}$. By (2.5), we find

$$
\sum_{m} L_{n} \epsilon_{k}(m) \omega(m)=\sum_{m} \omega(k) g(\tilde{k}, n, \tilde{m})=\sum_{m} \epsilon_{k}(m) \omega(m) \sum_{m} g(\tilde{k}, n, m) .
$$

Hence, $\omega$ is a Haar measure if and only if $\mathcal{K}$ is normalized.

In order to develop their theory further, Obata and Wildberger took care of the functional $\phi_{0}: \mathcal{A}_{0} \rightarrow \mathbb{C}$ defined by $\phi_{0}\left(\sum_{n} \alpha_{n} c_{n}\right)=\alpha_{0}$, and focused on the following property. A generalized hypergroup $\left(\mathcal{K}, \mathcal{A}_{0}\right)$ is said to satisfy property $(\mathrm{B})$ if for all $n$ there exists $\kappa(n) \geq 0$ such that $\left|\phi_{0}\left(b^{*} c_{n} b\right)\right| \leq \kappa(n) \phi_{0}\left(b^{*} b\right)$ for all $b \in \mathcal{A}_{0}$. We focus on a stronger property than Obata and Wildberger.

DEFINITION 2.6. A generalized hypergroup $\left(\mathcal{K}, \mathcal{A}_{0}\right)$ is called a semi-bounded generalized hypergroup (SBG hypergroup) if, additionally, the following axiom is valid.

(A4) For the structure constants, it is true that

$$
\gamma(n)=\sup _{m} \sum_{k}|g(n, m, k)|<\infty \quad \text { for all } n .
$$

A generalized hypergroup is called bounded if it is semi-bounded and $\gamma$ is bounded.

An SBG hypergroup satisfies property (B) with $\kappa(n)=\gamma(n)$, see [12, Theorem 4.1]. Then $\gamma(n) \geq \max \left(h(\tilde{n})^{-1}, 1\right)$. By straightforward arguments we have that

$$
\gamma(\tilde{m})=\sup _{n} \sum_{k}|g(n, m, k)| .
$$

If $\mathcal{K}$ is hermitian or commutative, then $\gamma(\tilde{n})=\gamma(n)$. If $\mathcal{K}$ is positive and normalized, then $\gamma(n)=1$ for all $n$. 


\section{Convolution of measures and functions}

Clearly, both measures and functions on $K$ can be identified with sequences indexed by $K$. However, we make a distinction anyway, since the natural definition of a convolution is different for measures and functions.

For discrete complex measures $\mu, \nu$ on $K$, we define a convolution by

$$
(\mu * \nu)(k)=\sum_{n, m} g(n, m, k) \mu(n) \nu(m)
$$

whenever the sum on the right-hand side is finite for all $k$. A short calculation shows $\epsilon_{0} * \mu=\mu * \epsilon_{0}=\mu$, that is, $\epsilon_{0}$ is the unit element for this convolution. For two Dirac measures we get $\epsilon_{n} * \epsilon_{m}=\sum_{k} g(n, m, k) \epsilon_{k}$, and supp $\epsilon_{n} * \epsilon_{m}$ is finite.

In order to investigate the convergence of the sum in (3.1) we introduce the spaces

$$
\begin{aligned}
M(K) & =\left\{\mu \text { measure on } K,|\mu|(K)=\sum_{n}|\mu(n)|<\infty\right\}, \quad\|\mu\|=|\mu|(K), \\
M_{\gamma}(K) & =\left\{\mu \in M(K),|\gamma \mu|(K)=\sum_{n}|\mu(n)| \gamma(n)<\infty\right\}, \quad\|\mu\|_{\gamma}=|\gamma \mu|(K) .
\end{aligned}
$$

The space $M_{\bar{\gamma}}(K)$ and the norm $\|\mu\|_{\bar{\gamma}}$ is defined analogously.

LEMMA 3.1. (i) If $\mu \in M_{\gamma}(K)$ and $\nu \in M(K)$, then $\mu * \nu \in M(K)$ and $\|\mu * v\| \leq\|\mu\|_{\gamma}\|v\|$.

(ii) If $\mu \in M(K)$ and $\nu \in M_{\tilde{\gamma}}(K)$, then $\mu * \nu \in M(K)$ and $\|\mu * \nu\| \leq\|\mu\|\|\nu\|_{\bar{\gamma}}$.

ProOF. (i) By Fubini,

$$
\|\mu * \nu\| \leq \sum_{n, m} \sum_{k}|g(n, m, k)\|\mu(n)\| \nu(m)| \leq \sum_{n, m} \gamma(n)\left|\mu(n)\|\nu(m) \mid=\| \mu\left\|_{\gamma}\right\| \nu \| .\right.
$$

The proof of (ii) is analogous using (2.9).

LEMMA 3.2. The convolution $*$ is associative, that is, $(\mu * v) * \rho=\mu *(\nu * \rho)$ whenever both expressions exist in the sense of Lemma 3.1.

PROOF. It suffices to prove the associativity for Dirac measures $\left(\epsilon_{n} * \epsilon_{m}\right) * \epsilon_{l}=$ $\epsilon_{n} *\left(\epsilon_{m} * \epsilon_{l}\right)$ For that purpose we use (2.6).

LEMMA 3.3. (i) It holds that $\left(\epsilon_{n} * \epsilon_{m}\right)^{-}=\overline{\epsilon_{\dot{m}} * \epsilon_{\tilde{n}}}$.

(ii) It holds that $0 \in \operatorname{supp} \epsilon_{n} * \epsilon_{\tilde{m}}$ if and only if $n=\tilde{m}$

(iii) If $\mathcal{K}$ is normalized, then $\epsilon_{n} * \epsilon_{m}(K)=1$ for all $n, m$.

(iv) It holds that $\epsilon_{n} * \epsilon_{\tilde{n}}(0)=h(\tilde{n})^{-1}>0$.

(v) It holds that $\left\|\epsilon_{n} * \epsilon_{m}\right\| \leq \min (\gamma(n), \gamma(\tilde{m}))$. 
ProOF. Using (2.5) we obtain (i), and application of axiom (A3) gives (ii). For (v) we have by definition $\left\|\epsilon_{n} * \epsilon_{m}\right\|=\sum_{k}|g(n, m, k)| \leq \gamma(n)$. The second inequality is achieved analogously, using (2.9). The assertions (iii) and (iv) are clear.

Now, we are able to compare the concept of an SBG hypergroup with that of a discrete hypergroup, see for example [5], or a discrete signed hypergroup, see [13]. Our previous results yield the following theorem.

THEOREM 3.4. (i) If $\mathcal{K}$ is a real, normalized and bounded generalized hypergroup, then its index set $K$ with convolution $*$ as defined in (3.1) and involution as defined in (2.1) is a discrete signed hypergroup.

(ii) If $\mathcal{K}$ is a positive and normalized $S B G$ hypergroup, then its index set $K$ with convolution $*$ and involution $\sim$ is a discrete hypergroup.

(iii) Let $(K, \star, \sim)$ be a discrete signed hypergroup. Put $\mathcal{K}=\left\{\epsilon_{k}, k \in K\right\}$ and let $\mathcal{A}_{0}$ be the vector space of all finite linear combinations of Dirac measures $\epsilon_{k} \in \mathcal{K}$. Further, let $\star$ be the multiplication in $\mathcal{A}_{0}$ and put $\epsilon_{k}^{*}=\epsilon_{\tilde{k}}$ as involution on $\mathcal{K}$, which is linearly extended to $\mathcal{A}_{0}$. Then $\left(\mathcal{K}, \mathcal{A}_{0}\right)$ is a real, bounded and normalized generalized hypergroup.

(iv) If $(K, *, \sim)$ is a discrete hypergroup, then the construction in (iii) yields a positive and normalized SBG hypergroup.

Next let us introduce the convolution of functions.

DEFINITION 3.5. Let $f$ and $g$ be functions on $K$ with finite support. The convolution of $f$ and $g$ is defined by

$$
(f \star g)(m)=\sum_{n} f(n)\left(\overline{L_{\tilde{n}}} g\right)(m) h(n) .
$$

LEMMA 3.6. If $f$ and $g$ have finite support, then $f \star g$ has finite support.

PROOF. By definition $(f \star g)(m)=\sum_{n, k} f(n) \overline{g(\tilde{n}, m, k)} g(k) h(n)$. Hence,

$$
\operatorname{supp} f \star g \subset \bigcup_{\substack{n \in \operatorname{supp} f \\ k \in \operatorname{supp} g}} M_{\bar{n}, k},
$$

with $M_{n, k}=\{m, g(n, m, k) \neq 0\}$. By [12, Lemma 1.2], the set $M_{n, k}$ is finite for all $n, k$.

For a function $a$ and a discrete measure $\mu$ on $K$ we denote the application of $\mu$ to $a$ by $\mu(a)=\sum_{k} a(k) \mu(k)$ whenever the sum exists. Furthermore, for a function $f$ and a measure $\mu$ we form the measure $f \mu$ by $f \mu(a)=\mu(f a)$ for all functions $a$ on $K$. 
THEOREM 3.7. If $f, g$ are functions on $K$ with finite support, then $(f \star g) h=$ $(f h) *(g h)$.

PROOF. Let $a$ be an arbitrary function on $K$. Application of Lemma 2.3 yields

$$
\begin{aligned}
(f \star g) h(a) & =\sum_{m} a(m)(f \star g)(m) h(m) \\
& =\sum_{m} \sum_{n} a(m) f(n)\left(\overline{L_{n}} g\right)(m) h(m) h(n) \\
& =\sum_{n} \sum_{m} f(n)\left(L_{n} a\right)(m) g(m) h(m) h(n) \\
& =\sum_{k} \sum_{m, n} g(n, m, k) f(n) h(n) g(m) h(m) a(k) \\
& =\sum_{k}(f h) *(g h)(k) a(k)=(f h) *(g h)(a) .
\end{aligned}
$$

If $\mathcal{K}$ is commutative, then $*$ is commutative and by the last lemma we see that $\star$ is then also commutative.

For a positive discrete measure $\sigma$ on $K$ and $1 \leq p<\infty$, we introduce the Banach spaces

$$
\begin{aligned}
l^{p}(\sigma) & =\left\{f: K \rightarrow \mathbb{C}, \sum_{n}|f(n)|^{p} \sigma(n)<\infty\right\}, & & \|f\|_{p, \sigma}=\left(\sum_{n}|f(n)|^{p} \sigma(n)\right)^{1 / p}, \\
l^{\infty} & =\left\{f: K \rightarrow \mathbb{C}, \sup _{n}|f(n)|<\infty\right\}, & & \|f\|_{\infty}=\sup _{n}|f(n)| .
\end{aligned}
$$

LEMMA 3.8. If $f \in l^{\infty}$, then $L_{n} f \in l^{\infty}$ for all $n$ and $\left\|L_{n} f\right\|_{\infty} \leq \gamma(n)\|f\|_{\infty}$.

PROOF. For all $n, m$,

$$
\left|L_{n} f(m)\right|=\left|\sum_{k} g(n, m, k) f(k)\right| \leq \sum_{k}\left|g(n, m, k)\|f f(k) \mid \leq \gamma(n)\| f \|_{\infty} .\right.
$$

We now see that the sums in (2.7) converge if $f \in l^{1}(h), g \in l^{\infty}$ or $f \in l^{\infty}$, $g \in l^{1}(h)$, respectively, and Lemma 2.3 extends to these spaces.

THEOREM 3.9. The convolution $\star$ in (3.2) extends to $l^{1}(\gamma h) \times l^{1}(h)$ and $\|f \star g\|_{1, h} \leq$ $\|f\|_{1, \gamma h}\|g\|_{1, h}$.

ProOF. First, assume $f, g$ have finite support and $a$ is such that

$$
a(k)(f \star g)(k)=|(f \star g)(k)| .
$$


Theorem 3.7 yields

$$
\begin{aligned}
\|(f \star g)\|_{1, h} & =\sum_{k}|(f \star g)(k)| h(k)=(f \star g) h(a)=|(f h) *(g h)(a)| \\
& \leq \sum_{n, m} \gamma(n)\left|g(m)\|f(n) \mid h(n) h(m)=\| f\left\|_{1, \gamma h}\right\| g \|_{1, h} .\right.
\end{aligned}
$$

Hence, $\star$ is continuous on a dense subspace of $l^{1}(\gamma h) \times l^{1}(h)$. Therefore, it can be uniquely continued.

Using (2.9), the convolution extends analogously to $l^{1}(h) \times l^{1}(\tilde{\gamma} h)$ with $\|f \star g\|_{1, h} \leq$ $\|f\|_{1, h}\|g\|_{1 . \tilde{\gamma} h}$. If $\mathcal{K}$ is bounded, that is, $\gamma(n) \leq M$ for all $n$, then the last theorem gives $\|f \star g\|_{1, h} \leq M\|f\|_{1, h}\|g\|_{1, h}$. For $f \in l^{1}(h)$, define $L_{f} g=f \star g$. Clearly, $L_{f}$ is a bounded operator on $l^{1}(h)$ and $\left\|L_{f}\right\| \leq M\|f\|_{1, h}$. With the norm $\|f\|^{\prime}=\left\|L_{f}\right\|$, it holds that $\|f \star g\|^{\prime} \leq\|f\|^{\prime}\|g\|^{\prime}$. Hence, if $\mathcal{K}$ is bounded, then $\left(l^{\prime}(h),\|\cdot\|^{\prime}, \star\right)$ is a Banach algebra.

LEMMA 3.10. For all $f \in l^{1}(h)$ and all $m, n \in K$,

$$
\left(L_{n} f\right)(m)=h(\tilde{n})^{-1} \overline{\left(\epsilon_{\tilde{n}} \star \bar{f}\right)(m)}
$$

and $\left\|L_{n} f\right\|_{1, h} \leq \gamma(\tilde{n})\|f\|_{1, h}$.

PROOF. Since $f \in l^{1}(h)$, the right-hand side of (3.3) exists by Theorem 3.9 and

$$
h(\tilde{n})^{-1} \overline{\left(\epsilon_{\tilde{n}} \star \bar{f}\right)(m)}=h(\tilde{n})^{-1} \sum_{k} \epsilon_{\tilde{n}}(k)\left(L_{\tilde{k}} f\right)(m) h(k)=\left(L_{n} f\right)(m) .
$$

By Theorem 3.9, we further deduce that

$$
\begin{aligned}
\left\|L_{n} f\right\|_{1, h} & =h(\tilde{n})^{-1}\left\|\overline{\epsilon_{\tilde{n}} \star \bar{f}}\right\|_{1, h} \leq h(\tilde{n})^{-1}\left\|\epsilon_{\tilde{n}}\right\|_{1, \gamma h}\|f\|_{1, h} \\
& =h(\tilde{n})^{-1} \sum_{k} \epsilon_{\tilde{n}}(k) \gamma(k) h(k)\|f\|_{1, h}=\gamma(\tilde{n})\|f\|_{1, h} .
\end{aligned}
$$

THEOREM 3.11. The convolution $\star$ in (3.2) extends to $l^{1}(\tilde{\gamma} h) \times l^{\infty}$, and

$$
\|f \star g\|_{\infty} \leq\|f\|_{1, \tilde{\gamma} h}\|g\|_{\infty} .
$$

Proof. Assume $f, g$ have finite support. By Lemma 3.8, we obtain

$$
\begin{aligned}
|(f \star g)(m)| & =\left|\sum_{n} f(n) \overline{\left(L_{\bar{n}} \bar{g}\right)(m)} h(n)\right| \\
& \leq \sum_{n}|f(n)| \gamma(\tilde{n}) h(n)\|g\|_{\infty}=\|f\|_{1, \tilde{y} h}\|g\|_{\infty} .
\end{aligned}
$$

Hence, $\star$ is bounded on a dense subspace of $l^{1}(\tilde{\gamma} h) \times l^{\infty}$ and can be extended. 
By $(f \star g)(m)=\sum_{n}\left(L_{\tilde{n}} \tilde{f}\right)(m) \tilde{g}(n) h(n)$, we prove analogously that the convolution extends to $l^{\infty} \times l^{1}(\gamma \tilde{h})$ with $\|f \star g\|_{\infty} \leq\|f\|_{\infty}\|g\|_{1, \gamma \bar{h}}$.

THEOREM 3.12. For $1 \leq p \leq \infty$, the convolution $\star$ in (3.2) extends to $\left(l^{1}(\gamma h) \cap\right.$ $\left.l^{1}(\tilde{\gamma} h)\right) \times l^{p}(h)$. With $1 / p+1 / q=1$,

$$
\|f \star g\|_{p, h} \leq\|f\|_{1, \gamma h}^{1 / p}\|f\|_{1, \tilde{y} h}^{1 / q}\|g\|_{p, h} .
$$

If $\mathcal{K}$ is hermitian or commutative, then (3.4) simplifies to $\|f \star g\|_{p, h} \leq\|f\|_{1, \gamma h}\|g\|_{p, h}$.

PROOF. For $f \in l^{1}(\gamma h) \cap l^{1}(\tilde{\gamma} h)$ put $L_{f} g=f \star g$. By Theorem 3.9, $\left\|L_{f}\right\|_{B\left(l^{1}(h)\right)} \leq$ $\|f\|_{1, \gamma h}$ where $B\left(l^{1}(h)\right)$ denotes the Banach space of bounded operators from $l^{1}(h)$ into $l^{1}(h)$. Furthermore, by Theorem 3.11 we have $\left\|L_{f}\right\|_{B\left(l^{x}\right)} \leq\|f\|_{1, \tilde{y} h}$. Hence, inequality (3.4) is a consequence of the Riesz-Thorin interpolation theorem, see for example [16, page 72]. If $\mathcal{K}$ is commutative, then $h=\tilde{h}$ and $\gamma=\tilde{\gamma}$.

By defining an operator $R_{g} f=f \star g$, we derive analogously that the convolution $\star$ extends to $l^{p}(h) \times\left(l^{1}(\tilde{\gamma} h) \cap l^{1}(\gamma \tilde{h})\right)$ with $\|f \star g\|_{p, h} \leq\|f\|_{p, h}\|g\|_{1, \tilde{\gamma} h}^{1 / p}\|g\|_{1, \gamma \tilde{h}}^{1 / q}$.

LEMMA 3.13. For $1 \leq p \leq \infty$ and $(1 / p+1 / q=1)$,

$$
\left\|L_{n} f\right\|_{p, h} \leq \gamma(\tilde{n})^{1 / p} \gamma(n)^{1 / q}\|f\|_{p, h} .
$$

PROOF. The proof uses (3.3) and Theorem 3.12.

THEOREM 3.14. Let $1 / p+1 / q=1$. For $f \in l^{p}(h), g \in l^{q}(h)$,

$$
|(f \star g)(m)| \leq \gamma(\tilde{m})^{1 / p} \gamma(m)^{1 / q}\|f\|_{p, h}\|\tilde{g}\|_{q, h} .
$$

PROOF. Applying Hölder's inequality in the second equation yields

$$
\begin{aligned}
|(f \star g)(m)| & =\left|\sum_{n} f(n) L_{\tilde{m}} \tilde{g}(n) h(n)\right|=\|f\|_{p, h}\left\|L_{\tilde{m}} \tilde{g}\right\|_{q, h} \\
& \leq \gamma(\tilde{m})^{1 / p} \gamma(m)^{1 / q}\|f\|_{p, h}\|\tilde{g}\|_{q, h} .
\end{aligned}
$$

If $\mathcal{K}$ is hermitian or commutative, inequality (3.5) becomes $|(f \star g)(m)| \leq$ $\gamma(m)\|f\|_{p, h}\|g\|_{q, h}$. In this case we introduce the Banach space

$$
l^{\infty}(\gamma)=\left\{f: K \rightarrow \mathbb{C}, \sup _{n} \frac{|f(n)|}{\gamma(n)}<\infty\right\}, \quad\|f\|_{\infty, \gamma}=\sup _{n} \frac{|f(n)|}{\gamma(n)} .
$$

Now, (3.5) becomes $\|f * g\|_{\infty, r} \leq\|f\|_{p, h}\|g\|_{q, h}$. 


\section{Dual objects}

We say that a generalized hypergroup $\left(\mathcal{K}^{\prime}, \mathcal{A}_{0}^{\prime}\right)$ is a function realization, if $\mathcal{A}_{0}^{\prime}$ is a dense subalgebra of the space $C(\mathcal{S})$, where $\mathcal{S}$ is a compact Hausdorff space. Using Gelfand theory, Obata and Wildberger proved that, for commutative generalized hypergroups $\left(\mathcal{K}, \mathcal{A}_{0}\right)$ satisfying (B), there is an isomorphism $a \rightarrow a^{\prime}$ onto a function realization $\left(\mathcal{K}^{\prime}, \mathcal{A}_{0}^{\prime}\right)$. Moreover, there is a positive Radon measure $\mu$ on $\mathcal{S}$ with $\operatorname{supp} \mu=S, \mu(\mathcal{S})=1$ and

$$
\phi_{0}(a)=\int_{\mathcal{S}} a^{\prime}(x) d \mu(x) \text { for all } a \in \mathcal{A}_{0},
$$

and $\mathcal{K}^{\prime}$ is a complete orthogonal set for $L^{2}(\mathcal{S}, \mu)$, see [12, Theorem 5.1].

From now on, we assume $\left(\mathcal{K}, \mathcal{A}_{0}\right)$ to be commutative and $\mathcal{A}_{0}$ to be a dense subalgebra of $C(\mathcal{S})$ for some compact Hausdorff space $\mathcal{S}$. Condition (B) now reads

$$
\left.\left.\left|\int_{\mathcal{S}} c_{n}(x)\right| b(x)\right|^{2} d \mu(x)\left|\leq \kappa(n) \int_{\mathcal{S}}\right| b(x)\right|^{2} d \mu(x)=\kappa(n)\|b\|_{L^{2}(\mathcal{S}, \mu)}^{2}
$$

for all $b \in C(\mathcal{S})$, and therefore, with $\kappa(n)=\left\|c_{n}\right\|_{\infty}=\sup _{x \in \mathcal{S}}\left|c_{n}(x)\right|<\infty$, condition (B) is satisfied. The next lemma states that $\kappa(n)$ cannot be chosen smaller.

LEMMA 4.1. Let $\left(\mathcal{K}, \mathcal{A}_{0}\right)$ satisfy condition (B) with constants $\kappa(n)$. Then

$$
\sup _{x \in \mathcal{S}}\left|c_{n}(x)\right| \leq \kappa(n) \quad \text { for all } n \in K .
$$

In particular, $\sup _{x \in \mathcal{S}}\left|c_{n}(x)\right| \leq \gamma(n)$.

PROOF. Let us first remark that $L^{2}(S, \mu)$ is the completion of $\mathcal{A}_{0}$ with respect to $\|\cdot\|_{2, \mu}$, since $\mathcal{K}$ is a complete orthogonal set for $L^{2}(\mathcal{S}, \mu)$. The inequality

$$
\left.\left.\left|\int_{\mathcal{S}} c_{n}(x)\right| b(x)\right|^{2} d \mu(x)\left|\leq \kappa(n) \int_{\mathcal{S}}\right| b(x)\right|^{2} d \mu(x)
$$

is hence valid even for all $b \in L^{2}(\mathcal{S}, \mu)$. Now, let $x_{0} \in \mathcal{S}$ and choose a family of neighborhoods $\left(V_{i}\right)_{i \in I}$ of $x_{0}$ such that $V_{i} \rightarrow\left\{x_{0}\right\}$. Further, let $b_{i}=\chi_{v_{i}} /\left\|\chi_{v_{i}}\right\|_{2, \mu}$ where $\chi_{v_{i}}$ denotes the characteristic function of the set $V_{i}$. Clearly $\lim _{i} \int_{\mathcal{S}} c_{n}(x)\left|b_{i}(x)\right|^{2} d \mu(x)=$ $c_{n}\left(x_{0}\right)$. Since, $x_{0} \in \mathcal{S}$ is arbitrarily chosen, inserting into (4.1) gives the assertion. Further, $\kappa(n)=\gamma(n)$ is a valid choice by [12, Theorem 4.1].

Let us now consider dual objects of commutative generalized hypergroups. Obata and Wildberger already have defined characters [12, page 74], but their definition seems to be too weak in order to develop harmonic analysis. 
DEFINITION 4.2. We define two dual spaces by

$$
\begin{aligned}
\mathcal{X}^{b}(K) & =\left\{\alpha \in l^{\infty}(\gamma), \alpha \neq 0, L_{n} \alpha(m)=\alpha(n) \alpha(m)\right\}, \\
\hat{K} & =\left\{\alpha \in \mathcal{X}^{b}(K), \alpha(\tilde{n})=\overline{\alpha(n)}\right\} .
\end{aligned}
$$

The elements of $\mathcal{X}^{b}(K)$ are called characters and the elements of $\hat{K}$ hermitian characters.

Consider an element $x$ of $\mathcal{S}$. It can be seen that $\alpha_{x}(n)=c_{n}(x)$ defines an element of $\hat{K}$. Hence $\hat{K} \neq \emptyset$. Since $\mathcal{A}_{0}$ is dense in $C(\mathcal{S})$ and $\mathcal{S}$ is a compact Hausdorff space, it follows that for different $x, y \in \mathcal{S}$ we obtain different characters $\alpha_{x} \neq \alpha_{y}$, see also [12, Theorem 6.4]. Thus, we can identify $\mathcal{S}$ with a subset of $\hat{K}$ and we get the inclusion relations

$$
\mathcal{S} \subset \hat{K} \subset \mathcal{X}^{b}(K)
$$

The latter relation is well known for hypergroups and signed hypergroups. In contrast. to the group case, these inclusions may be proper, as is illustrated by some known examples for hypergroups.

From $\alpha(n)=L_{0} \alpha(n)=\alpha(0) \alpha(n)$, it follows that $\alpha(0)=1$. Furthermore, since $\gamma(0)=1,\|\alpha\|_{\infty, \gamma} \geq 1$. By Lemma $4.1,\left|c_{n}(x)\right| \leq \gamma(n)$, which implies $\left\|\alpha_{x}\right\|_{\infty, \gamma}=1$ for all $x \in \mathcal{S}$. For $r \geq 1$ let us define the following subsets of the duals

$$
\mathcal{X}_{r}^{b}(K)=\left\{\alpha \in \mathcal{X}^{b}(K),\|\alpha\|_{\infty, \gamma} \leq r\right\}, \quad \hat{K}_{r}=\left\{\alpha \in \hat{K},\|\alpha\|_{\infty, \gamma} \leq r\right\} .
$$

If $\mathcal{K}$ is bounded, then $\mathcal{X}^{b}(K)=\mathcal{X}_{R}^{b}(K)$ and $\hat{K}=\hat{K}_{R}$, where $R=\sup _{n} \gamma(n) \leq \infty$. In fact, in that case $l^{\infty}(\gamma)=l^{\infty}$ setwise and for a character $\alpha \in l^{\infty}$,

$$
|\alpha(n)|^{2}=|\alpha(n) \alpha(n)|=\left|L_{n} \alpha(n)\right| \leq \gamma(n)\|\alpha\|_{\infty} .
$$

Taking the supremum over all $n \in K$ yields $\|\alpha\|_{\infty} \leq \sup _{n} \gamma(n)$. Since $\gamma(n) \geq 1$, we further deduce that

$$
1 \leq\|\alpha\|_{\infty, \gamma} \leq\|\alpha\|_{\infty} \leq R
$$

We equip $\mathcal{X}^{b}(K)$ with the topology of pointwise convergence and subsets of $\mathcal{X}^{b}(K)$ with the induced topologies. With these topologies the functions $s_{n}: \mathcal{X}^{b}(K) \rightarrow \mathbb{C}$, $s_{n}(\alpha)=\alpha(n)$ and their restrictions to the other duals are continuous. We state without proof that the Gelfand topology on $\mathcal{S}$ is the topology induced by $\mathcal{X}^{b}(K)$, that is, the topology of pointwise convergence. 


\section{Fourier transform}

Due to our dual objects, we are able to perform some Fourier analysis in the context of commutative SBG hypergroups.

DEFINITION 5.1. For $\mu \in M_{\gamma}(K)$ we introduce the following two versions of the Fourier-Stieltjes-transform

$$
\begin{aligned}
& \hat{\mu}(\alpha)=\sum_{n} \alpha(n) \mu(n) \quad \text { for } \alpha \in \hat{K}, \\
& \mathcal{F}(\mu)(\alpha)=\sum_{n} \alpha(n) \mu(n) \quad \text { for } \alpha \in \mathcal{X}^{b}(K) \text {. }
\end{aligned}
$$

For $x \in \mathcal{S} \subset \hat{K}$, we write $\hat{\mu}(x)=\hat{\mu}\left(\alpha_{x}\right)=\sum_{n} c_{n}(x) \mu(n)$.

The following lemma states that our definition makes sense.

LEMMA 5.2. If $\alpha \in \mathcal{X}_{r}^{b}(K)$, then $|\mathcal{F}(\mu)(\alpha)| \leq r\|\mu\|_{\gamma}$ and $\mathcal{F}(\mu)$ is a continuous function from $\mathcal{X}_{r}^{b}(K)$ into $\mathbb{C}$.

PROOF. Let $\alpha \in \mathcal{X}_{r}^{b}(K)$, that is, $|\alpha(n)| \leq r \gamma(n)$ for all $n$. We obtain

$$
|\mathcal{F}(\mu)(\alpha)| \leq \sum_{n}\left|\alpha(n)\left\|\mu(n)\left|\leq r \sum_{n}\right| \gamma(n)\right\| \mu(n)\right|=r\|\mu\|_{\gamma} .
$$

Since the functions $s_{n}(\alpha)=\alpha(n)$ are continuous on $\mathcal{X}_{r}^{b}(K)$ for fixed $n$, it follows that $\mathcal{F}(\mu)$ is continuous on $\mathcal{X}_{r}^{b}(K)$.

DEFINITION 5.3. For $f \in l^{1}(\gamma h)$ we define two versions of the Fourier transform by

$$
\begin{array}{cc}
\hat{f}(\alpha)=\widehat{(f h)}(\alpha)=\sum_{n} f(n) \alpha(n) h(n) & \text { for } \alpha \in \hat{K}, \\
\mathcal{F}(f)(\alpha)=\mathcal{F}(f h)(\alpha)=\sum_{n} f(n) \alpha(n) h(n) & \text { for } \alpha \in \mathcal{X}^{b}(K) .
\end{array}
$$

For $x \in \mathcal{S}$, we write $\hat{f}(x)=\hat{f}\left(\alpha_{x}\right)=\sum_{n} f(n) c_{n}(x) h(n)$.

By interpreting measures on $K$ as functions on $K$ we clearly have $l^{1}(\gamma h)=$ $\left\{f, f h \in M_{\gamma}(K)\right\}$ and hence, Lemma 5.2 immediately implies that the Fourier transform is continuous on $\mathcal{X}_{r}^{b}(K)$ for all $r \geq 1$ and that for $\alpha \in \mathcal{X}_{r}^{b}(K)$,

$$
|\hat{f}(\alpha)| \leq\|f\|_{1, \gamma h} .
$$


In order to define the Fourier transform for $f \in l^{2}(h)$ we note that $\left\{\sqrt{h(n)} c_{n}, n \in K\right\}$ is a complete orthonormal set for $L^{2}(\mathcal{S}, \mu)$, see [12, Corollary 3.4]. Therefore, the series $\sum_{n} f(n) c_{n} h(n)$ converges in $L^{2}(\mathcal{S}, \mu)$ by Parseval's identity

$$
\begin{aligned}
\int_{\mathcal{S}}\left|\sum_{n} f(n) c_{n}(x) h(n)\right|^{2} d \mu(x) & =\left\|\sum_{n} f(n) c_{n} h(n)\right\|_{2, \mu}^{2} \\
& =\sum_{n}|f(n)|^{2} h(n)=\|f\|_{2, h}^{2} .
\end{aligned}
$$

Hence, we define the Fourier transform of $f \in l^{2}(h)$ by $\hat{f}=\sum_{n} f(n) c_{n} h(n)$, where convergence of the sum is understood in $L^{2}(\mathcal{S}, \mu)$. In (5.2) we already proved Plancherel's theorem.

THEOREM 5.4. The Fourier transform is an isometric isomorphism from $l^{2}(h)$ into $L^{2}(\mathcal{S}, \mu)$. In particular, for $f \in l^{2}(h),\|\hat{f}\|_{2, \mu}=\|f\|_{2 . h}$.

As a consequence of Plancherel's theorem we obtain a uniqueness theorem for the Fourier transform on $l^{1}(\gamma h)$.

THEOREM 5.5. If $f \in l^{\prime}(\gamma h)$ and $\mathcal{F}(f)_{\mid \mathcal{S}}=0$, then $f=0$.

PROOF. Let $f \in l^{1}(\gamma h)$. Since $\gamma(n) \geq 1$ we have $f \in l^{1}(h)$. Set $N=\{n \in K$, $|f(n)| \geq 1\}$. Since $\gamma(n) \geq h(n)^{-1}$, this set is finite. We obtain

$$
\sum_{n \in K}|f(n)|^{2} h(n) \leq \sum_{n \in N}|f(n)|^{2} h(n)+\|f\|_{1, h}<\infty,
$$

which means $f \in l^{2}(h)$. The Fourier transform on $l^{1}(\gamma h)$ coincides with the one on $l^{2}(h) \mu$-almost everywhere and by Plancherel's theorem

$$
\|f\|_{2, h}=\|\hat{f}\|_{2, \mu}=\left\|\mathcal{F}(f)_{\mid \mathcal{S}}\right\|_{2, \mu}=0 .
$$

We therefore obtain $f=0$.

Let us turn our attention now to the relation of Fourier transform and convolution.

THEOREM 5.6. If $f, g \in l^{1}(\gamma h)$ such that $f \star g \in l^{1}(\gamma h)$, then

$$
\mathcal{F}(f \star g)(\alpha)=\mathcal{F}(f)(\alpha) \mathcal{F}(g)(\alpha) \text { for all } \alpha \in \mathcal{X}^{b}(K)
$$


ProOF. We use Lemma 2.3 and Fubini's theorem to obtain

$$
\begin{aligned}
\mathcal{F}(f \star g)(\alpha) & =\sum_{n}(f \star g)(n) \alpha(n) h(n) \\
& =\sum_{n} \sum_{m} f(m)\left(\overline{L_{\tilde{m}}} g\right)(n) h(m) \alpha(n) h(n) \\
& =\sum_{m} \sum_{n}\left(L_{m} \alpha\right)(n) g(n) h(n) f(m) h(m) \\
& =\sum_{m} \sum_{n} g(n) \alpha(n) h(n) f(m) \alpha(m) h(m) \\
& =\sum_{n} g(n) \alpha(n) h(n) \sum_{m} f(m) \alpha(m) h(m) \\
& =\mathcal{F}(f)(\alpha) \mathcal{F}(g)(\alpha) .
\end{aligned}
$$

COROLlaRY 5.7. The convolution $\star$ extends to $l^{1}(\gamma h) \times l^{1}(\gamma h) \rightarrow l^{2}(h)$. It holds that

$$
\|f \star g\|_{2, h} \leq\|f\|_{1, \gamma h}\|g\|_{1, \gamma h} .
$$

ProOF. First suppose $f, g \in l^{1}(\gamma h)$ such that $f \star g \in l^{1}(\gamma h)$. Using Plancherel's Theorem 5.4, Theorem 5.6 and (5.1), we obtain

$$
\|f \star g\|_{2, h}=\|\widehat{f \star g}\|_{2, \mu}=\|\hat{f} \hat{g}\|_{2, \mu} \leq\|\hat{f}\|_{\infty, \mathcal{S}}\|\hat{g}\|_{\infty, \mathcal{S}} \leq\|f\|_{1, \gamma h}\|g\|_{1, \gamma h} .
$$

Hence, the convolution is continuous on $C=\left\{(f, g), f, g \in l^{1}(\gamma h), f \star g \in l^{1}(\gamma h)\right\}$. Since functions of finite support are dense in $l^{l}(\gamma h)$ and the convolution of two such functions has again finite support, we see that $C$ is dense in $l^{1}(\gamma h) \times l^{1}(\gamma h)$. Thus $\star$ uniquely extends to $l^{1}(\gamma h) \times l^{1}(\gamma h)$ and (5.4) holds.

Implicitly we used the commutativity of $\mathcal{K}$ in this proof. Immediately, we obtain that the convolution Theorem 5.6 holds for all $f, g \in l^{1}(\gamma h)$ with the slight adjustment that in general (5.3) holds only for $\mu$-almost all $\alpha \in \mathcal{X}^{\prime \prime}(K)$.

An involution on $l^{1}(h)$ is given by $f^{*}(n)=\overline{f(\tilde{n})}$, which is preserved by the Fourier transform on $\hat{K}$, that is,

$$
\widehat{f^{*}}(\alpha)=\sum_{n} \overline{f(\tilde{n})} \alpha(n) h(n)=\sum_{n} \overline{f(\tilde{n}) \alpha(\tilde{n})} h(n)=\overline{\hat{f}(n)} \quad \text { for all } \alpha \in \hat{K} .
$$

The inverse Fourier transform for $F \in L^{1}(\mathcal{S}, \mu)$ is defined by

$$
\check{F}(n)=\int_{\mathcal{S}} F(x) \overline{c_{n}(x)} d \mu(x), \quad \text { for all } n \in K .
$$


We can even extend this definition to a larger space. Let $M(\mathcal{S})$ denote the space of complex bounded Radon measures on $\mathcal{S}$ with the total variation as norm. For $\rho \in M(\mathcal{S})$ we define the inverse Fourier-Stieltjes transform by

$$
\check{\rho}(n)=\int_{\mathcal{S}} \overline{c_{n}(x)} d \rho(x), \quad \text { for all } n \in K .
$$

Clearly, $(F \mu)^{\swarrow}=\check{F}$. Parseval's identity immediately gives the following inversion theorem.

THEOREM 5.8. (i) If $f \in l^{2}(h)$, then $(\hat{f})^{\swarrow}=f$.

(ii) If $F \in L^{2}(\mathcal{S}, \mu)$, then $\widehat{(\check{F})}=F \mu$-almost everywhere.

THEOREM 5.9. For the inverse Fourier-Stieltjes transform the following are true.

(i) For $\rho \in M(\mathcal{S})$ we have $\check{\rho} \in l^{\infty}(\gamma)$ and $\|\check{\rho}\|_{\infty, \gamma} \leq\|\rho\|$.

(ii) For $F \in L^{1}(\mathcal{S}, \mu)$ it holds that $\|\check{F}\|_{\infty, \gamma} \leq\|F\|_{1, \mu}$.

(iii) For $F \in L^{1}(\mathcal{S}, \mu)$ we have $\check{F} \in c_{0}(\gamma)$ where $c_{0}(\gamma)$ denotes the closure with respect to $\|\cdot\|_{\infty, \gamma}$ of the set of all functions with finite support. Furthermore, the image of the inverse Fourier transform of $L^{1}(\mathcal{S}, \mu)$ is dense in $c_{0}(\gamma)$.

ProOF. (i) For $\rho \in M(\mathcal{S})$ and $n \in K$ we have

$$
|\check{\rho}(n)|=\left|\int_{\mathcal{S}} \overline{c_{n}}(x) d \rho(x)\right| \leq\left\|c_{n}\right\|_{\infty, \mathcal{S}}\|\rho\| \leq \gamma(n)\|\rho\| .
$$

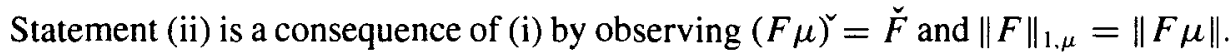

(iii) Let $\epsilon>0$ and choose $G \in C(\mathcal{S})$ such that $\|F-G\|_{1, \mu} \leq \epsilon / 2$. Since $C(\mathcal{S}) \subset L^{2}(\mathcal{S}, \mu)$, it holds that $\breve{G} \in l^{2}(h)$. Hence, there exists $\phi$ with $|\operatorname{supp} \phi|<\infty$ such that $\|\check{G}-\phi\|_{2, h} \leq \epsilon / 2$. Using $\gamma(n) \geq \max \left\{1, h(n)^{-1}\right\}$ we deduce, for arbitrary $f \in l^{2}(h)$, that

$$
\frac{|f(n)|^{2}}{\gamma(n)^{2}} \leq \frac{|f(n)|^{2}}{\gamma(n)} \leq|f(n)|^{2} h(n) \leq \sum_{n}|f(n)|^{2} h(n)=\|f\|_{2, h}^{2},
$$

yielding $\|f\|_{\infty, \gamma} \leq\|f\|_{2, h}$. We hereby derived $l^{2}(h) \subset l^{\infty}(\gamma)$. Now, using this estimation, we obtain $\|\check{G}-\phi\|_{\infty, \gamma} \leq\|\check{G}-\phi\|_{2, h} \leq \epsilon / 2$ and further

$$
\begin{aligned}
|\check{F}(k)-\phi(k)| & \leq|\check{F}(k)-\check{G}(k)|+|\check{G}(k)-\phi(k)| \\
& \leq \gamma(k)\left(\|F-G\|_{1, \mu}+\epsilon / 2\right) \leq \epsilon \gamma(k),
\end{aligned}
$$

which is equivalent to $\|\check{F}-\phi\|_{\infty, \gamma} \leq \epsilon$. Hence, $\check{F}$ can be approximated with respect to $\|\cdot\|_{\infty, y}$ by functions with finite support. Since all function with finite support are contained in the image of the inverse Fourier transform of $L^{1}(\mathcal{S}, \mu)$, the image of $L^{1}(\mathcal{S}, \mu)$ is dense in $c_{0}(\gamma)$. 
The last result generalizes the Riemann-Lebesgue lemma. We also have a uniqueness theorem for the inverse Fourier transform.

THEOREM 5.10. Let $\rho \in M(\mathcal{S})$. If $\check{\rho}=0$, then $\rho=0$.

ProOF. Assume that $\rho \neq 0$, but $\check{\rho}=0$. By [12, Theorem 5.1]

$$
\mathcal{A}_{0}=\left\{\hat{f}_{\mid \mathcal{S}},|\operatorname{supp} f|<\infty\right\}
$$

is a dense subalgebra of $C(\mathcal{S})$. Hence, there is some $f$ with finite support such that $\int_{\mathcal{S}} \hat{f}(x) d \rho(x) \neq 0$. However, we have

$$
\int_{\mathcal{S}} \hat{f}(x) d \rho(x)=\sum_{n} f(n) \int_{\mathcal{S}} \overline{c_{\tilde{n}}(x)} d \rho(x) h(n)=\sum_{n} f(n) \check{\rho}(\tilde{n}) h(n)=0 .
$$

Denoting $p_{n}(x)=\epsilon_{n}(x) / h(n)$, we have $\hat{p}_{n}(x)=c_{n}(x)$ and $\left(\hat{p}_{n}\right)^{\swarrow}=p_{n}$ yielding

$$
\check{\mu}(n)=\int_{\mathcal{S}} \overline{c_{n}(x)} d \mu(x)=p_{\tilde{n}}(0)=\epsilon_{0}(\tilde{n}),
$$

that is, $\check{\mu}=\epsilon_{0}$. Another important property was shown in the proof of Theorem 5.10 above. Suppose $f$ has finite support and $\rho \in M(\mathcal{S})$. Then

$$
\int_{\mathcal{S}} \hat{f}(x) d \rho(x)=\sum_{n} f(n) \check{\rho}(\tilde{n}) h(n) .
$$

We can extend the uniqueness theorem to the following result.

THEOREM 5.11. Let $f \in l^{1}(\gamma h)$ and $\rho \in M(\mathcal{S})$. Then $\check{\rho}=f$ if and only if $\rho=\hat{f} \mu$.

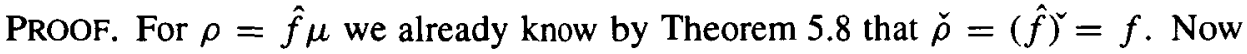
suppose $f=\check{\rho}$ and let $g$ have finite support. With (5.5) and $(\hat{g})^{2}=g$ we obtain

$$
\begin{aligned}
\int_{\mathcal{S}} \hat{g}(x) \hat{f}(x) d \mu(x) & =\int_{\mathcal{S}} \hat{g}(x) \sum_{n} f(n) c_{n}(x) h(n) d \mu(x) \\
& =\sum_{n} f(n) \int_{\mathcal{S}} \hat{g}(x) c_{n}(x) d \mu(x) h(n) \\
& =\sum_{n} f(n) \int_{\mathcal{S}} \hat{g}(x) \overline{c_{\tilde{n}}(x)} d \mu(x) h(n) \\
& =\sum_{n} f(n) g(\tilde{n}) h(n)=\sum_{n} g(n) \check{\rho}(\tilde{n}) h(n)=\int_{\mathcal{S}} \hat{g}(x) d \rho(x) .
\end{aligned}
$$

Since $\left\{\hat{g}_{\mid \mathcal{S}},|\operatorname{supp} g|<\infty\right\}$ is dense in $C(\mathcal{S})$, we see that $\rho=\hat{f} \mu$. 
A rewriting of the last result gives the inversion theorem.

THEOREM 5.12. The following two inversion formulae hold.

(i) Let $f \in l^{1}(\gamma h)$. Then for every $n \in K, f(n)=\int_{\mathcal{S}} \hat{f}(x) \overline{c_{n}(x)} d \mu(x)$.

(ii) Let $F \in L^{1}(\mathcal{S}, \mu)$ such that $\check{F} \in l^{1}(\gamma h)$. Then for $\mu$-almost every $x \in \mathcal{S}$,

$$
F(x)=\sum_{n} \check{F}(n) c_{n}(x) h(n) .
$$

If in addition $F$ is continuous, then (5.6) holds for all $x \in \mathcal{S}$.

PROOF. (i) follows by Theorem 5.8 (i). For (ii) put $\rho=F \mu$. Then $\check{\rho}=\check{F} \in l^{1}(\gamma h)$. By Theorem 5.11, we have $\rho=(\check{F})^{\hat{\mu}} \mu$, which is equivalent to $F=(\check{F})^{\wedge}$ in $L^{1}(\mathcal{S}, \mu)$. Since the right-hand side of (5.6) is continuous, equality holds for all $x \in \mathcal{S}$ if $F$ is continuous.

\section{Orthogonal polynomials on the real line}

Let $\mu$ be a probability measure on the real line. We denote the support of $\mu$ by $\mathcal{S}$ and assume $\operatorname{card}(\mathcal{S})=\infty$. Furthermore, let $\left(P_{n}\right)_{n=0}^{\infty}$ denote an orthogonal polynomial sequence with respect to $\mu$, that is $\int P_{n} P_{m} d \mu \neq 0$ if and only if $n=m$. The polynomials $P_{n}$ are assumed to have real coefficients with $\operatorname{deg}\left(P_{n}\right)=n$ and $P_{0}=1$. It is well-known that the sequence $\left(P_{n}\right)_{n \in \mathbb{N}_{0}}$ satisfies a three term recurrence relation of the following type

$$
P_{1}(x) P_{n}(x)=a_{n} P_{n+1}(x)+b_{n} P_{n}(x)+c_{n} P_{n-1}(x), \quad n \geq 1,
$$

with $P_{0}(x)=1$ and $P_{1}(x)=(x-b) / a$, where the coefficients are real numbers with $c_{1}>0, c_{n} a_{n-1}>0, n>1$. Conversely, if we define $\left(P_{n}\right)_{n=0}^{\infty}$ by $(6.1)$, there is a measure $\mu$ with the assumed properties, see [3].

The linearization coefficients $g(n, m, k)$ are uniquely defined by

$$
P_{n} P_{m}=\sum_{k=0}^{\infty} g(n, m, k) P_{k}=\sum_{k=|n-m|}^{n+m} g(n, m, k) P_{k} .
$$

The linearization coefficients are obtained recursively based on the coefficients of the three term recurrence relation. The corresponding formulae are written down in the following lemma for ease of reference.

LEMMA 6.1. We have $g(0, m, m)=1$. In case $m \geq 1, g(1, m, m-1)=c_{m}$, $g(1, m, m)=b_{m}$ and $g(1, m, m+1)=a_{m}$. In case $m \geq n \geq 2$, we get the recurrence relation: 
(i)

(6.3) $\quad g(n, m, n+m)=g(n-1, m, n+m-1) \frac{a_{n+m-1}}{a_{n-1}}=\frac{a_{m} a_{m+1} \cdots a_{n+m-1}}{a_{1} a_{2} \cdots a_{n-1}}$

$$
g(n, m, m-n)=g(n-1, m, m-n+1) \frac{c_{m-n+1}}{a_{n-1}}=\frac{c_{m} c_{m-1} \cdots c_{m-n+1}}{a_{1} a_{2} \cdots a_{n-1}},
$$

(ii) $g(n, m, n+m-1)=g(n-1, m, n+m-1) \frac{b_{n+m-1}-b_{n-1}}{a_{n-1}}$

$$
\begin{aligned}
& +g(n-1, m, n+m-2) \frac{a_{n+m-2}}{a_{n-1}}, \\
g(n, m, m-n+1)= & g(n-1, m, m-n+1) \frac{b_{m-n+1}-b_{n-1}}{a_{n-1}} \\
& +g(n-1, m, m-n+2) \frac{c_{m-n+2}}{a_{n-1}},
\end{aligned}
$$

(iii) For $k=2,3, \ldots, 2 n-2$,

$$
\begin{aligned}
g(n, m, m-n+k)= & g(n-1, m, m-n+k-1) \frac{a_{m-n+k-1}}{a_{n-1}} \\
& +g(n-1, m, m-n+k) \frac{b_{m-n+k}-b_{n-1}}{a_{n-1}} \\
& +g(n-1, m, m-n+k+1) \frac{c_{m-n+k+1}}{a_{n-1}} \\
& -g(n-2, m, m-n+k) \frac{c_{n-1}}{a_{n-1}}
\end{aligned}
$$

PROOF. In case $m \geq n \geq 2$, we have

$$
P_{n}=\frac{1}{a_{n-1}} P_{1} P_{n-1}-\frac{b_{n-1}}{a_{n-1}} P_{n-1}-\frac{c_{n-1}}{a_{n-1}} P_{n-2} .
$$

So

$$
\begin{aligned}
P_{n} P_{m}= & \sum_{k=m-n+1}^{m+n-1} g(n-1, m, k)\left(\frac{a_{k}}{a_{n-1}} P_{k+1}+\frac{b_{k}}{a_{n-1}} P_{k}+\frac{c_{k}}{a_{n-1}} P_{k-1}\right) \\
& -\frac{b_{n-1}}{a_{n-1}} \sum_{m-n+1}^{m+n-1} g(n-1, m, k) P_{k}-\frac{c_{n-1}}{a_{n-1}} \sum_{m-n+2}^{m+n-2} g(n-2, m, k) P_{k},
\end{aligned}
$$

which implies the recurrence formulae (i)-(iii). The second equations in (i) are proven by induction.

We easily derive

$$
h(n)=g(n, n, 0)^{-1}=\left(\int P_{n}^{2}(x) d \mu(x)\right)^{-1}=\frac{\prod_{i=1}^{n-1} a_{i}}{\prod_{i=1}^{n} c_{i}} .
$$


Let $\mathcal{K}=\left\{P_{n}, n \in \mathbb{N}_{0}\right\}, \mathcal{A}_{0}$ be the set of polynomials with complex coefficients in one real variable and ${ }^{*}$ be the complex conjugation ${ }^{-}$.

THEOREM 6.2. We have the following classification.

(i) $\left(\mathcal{K}, \mathcal{A}_{0}\right)$ is a hermitian and commutative generalized hypergroup.

(ii) $\left(\mathcal{K}, \mathcal{A}_{0}\right)$ satisfies property (B) if and only if $\mathcal{S}$ is compact. $\mathcal{S}$ is compact if and only if the sequences $\left(c_{n} a_{n-1}\right)$ and $\left(b_{n}\right)$ are bounded.

(iii) $\left(\mathcal{K}, \mathcal{A}_{0}\right)$ is an SBG hypergroup if and only if the sequences $\left(a_{n}\right),\left(b_{n}\right)$ and $\left(c_{n}\right)$ are bounded.

PROOF. For (i) and (ii) see [3] and [12].

If $\mathcal{K}$ is semi-bounded, then there is a bound for $g(1, n, n+1)=a_{n}, g(1, n, n)=b_{n}$ and $g(1, n, n-1)=c_{n}$. Let $\left|a_{n}\right|,\left|b_{n}\right|,\left|c_{n}\right|<B$. It is sufficient to prove $|g(n, m, k)|<$ $M_{n}$ for all $m, k \in \mathbb{N}_{0}$, which implies $\sum_{k=|n-m|}^{n+m}|g(n, m, k)| \leq(2 n+1) M_{n}$ for all $m \in \mathbb{N}_{0}$. Then $M_{0}=1$ and $M_{1}=B$ is a proper choice. Now let us assume that for $n \geq 2$ there exist proper $M_{0}, M_{1}, \ldots, M_{n-1}$. According to the recurrence relation of the linearization coefficients, see Lemma 6.1, we get

$$
|g(n, m, k)| \leq \frac{4 B}{\left|a_{n-1}\right|} M_{n-1}+\frac{B}{\left|a_{n-1}\right|} M_{n-2}=M_{n} .
$$

Therefore we call $\mathcal{K}$ a generalized polynomial hypergroup or an $S B G$ polynomial hypergroup, respectively. In order to get normalized generalized hypergroups, Obata and Wildberger have investigated renormalizations in [12]. The following lemma shows that there always exists a renormalization of a generalized polynomial hypergroup $\mathcal{K}=\left\{P_{n} ; n \in \mathbb{N}_{0}\right\}$ with property (B), which is semi-bounded.

LEMMA 6.3. Suppose $\mu$ has compact support $\mathcal{S}$. Then the monic polynomials $Q_{n}$ and the orthonormal polynomials $p_{n}=\sqrt{h(n)} P_{n}$ with respect to $\mu$ constitute an SBG polynomial hypergroup.

PROOF. Let the monic polynomials be defined by $Q_{0}=1, Q_{1}(x)=x-b^{\prime}$ and $Q_{1} Q_{n}=Q_{n+1}+b_{n}^{\prime} Q_{n}+c_{n}^{\prime} Q_{n-1}, n \geq 1$, where $c_{n}^{\prime}>0$. Since $\mu$ has compact support, $\left(b_{n}^{\prime}\right)$ and $\left(c_{n}^{\prime}\right)$ are bounded sequences. By Theorem 6.2 (iii), the corresponding generalized hypergroup is semi-bounded. Now, it is simple to derive that the corresponding orthonormal polynomials are defined by $p_{0}=1, p_{1}=\left(x-b^{\prime}\right) / \sqrt{c_{1}^{\prime}}$ and $p_{1} p_{n}=a_{n}^{\prime \prime} p_{n+1}+b_{n}^{\prime} / \sqrt{c_{1}^{\prime}} p_{n}+a_{n-1}^{\prime \prime} p_{n-1}$, where $a_{n}^{\prime \prime}=\sqrt{c_{n+1}^{\prime} / c_{1}^{\prime}}$. Since $\left(c_{n}^{\prime}\right)$ is bounded, by Theorem 6.2 (iii), the corresponding generalized hypergroup is semi-bounded.

Now, we are looking for an OPS $\left(R_{n}\right)_{n \in \mathbb{N}_{0}}$ with $\sum_{k} g^{R}(n, m, k)=1$ for all $n, m \in$ $\mathbb{N}_{0}$, which is equivalent to the existence of $x_{0} \in \mathbb{R}$ with $R_{n}\left(x_{0}\right)=1$ for all $n \in \mathbb{N}_{0}$. 
THEOREM 6.4. Suppose $\mu$ has compact support and let $\left(P_{n}\right)_{n \in \mathbb{N}_{0}}$ be an arbitrary orthogonal polynomial sequence with respect to $\mu$. Denote by $[d, e]$ the smallest interval containing $S$. Choose $x_{0} \in \mathbb{R} \backslash(d, e)$ and define $R_{n}(x)=P_{n}(x) / P_{n}\left(x_{0}\right)$, $n \in \mathbb{N}_{0}$. Then $\mathcal{K}=\left\{R_{k}, k \in \mathbb{N}_{0}\right\}$ is a normalized SBG hypergroup.

PROOF. Let $\left(Q_{n}\right)_{n \in \mathbb{N}_{0}}$ be the monic orthogonal polynomials with respect to $\mu$ as in the proof of Lemma 6.3. Then

$$
R_{1} R_{n}=\frac{Q_{n+1}\left(x_{0}\right)}{Q_{1}\left(x_{0}\right) Q_{n}\left(x_{0}\right)} R_{n+1}+\frac{b_{n}^{\prime}}{Q_{1}\left(x_{0}\right)} R_{n}+\frac{c_{n}^{\prime} Q_{n-1}\left(x_{0}\right)}{Q_{1}\left(x_{0}\right) Q_{n}\left(x_{0}\right)} R_{n-1}, \quad n \geq 1
$$

Let

$$
a_{n}^{\prime \prime \prime}=\frac{Q_{n+1}\left(x_{0}\right)}{Q_{1}\left(x_{0}\right) Q_{n}\left(x_{0}\right)}, \quad b_{n}^{\prime \prime \prime}=\frac{b_{n}^{\prime}}{Q_{1}\left(x_{0}\right)} \quad \text { and } \quad c_{n}^{\prime \prime \prime}=\frac{c_{n}^{\prime} Q_{n-1}\left(x_{0}\right)}{Q_{1}\left(x_{0}\right) Q_{n}\left(x_{0}\right)}
$$

Since $\left(b_{n}^{\prime}\right)$ is bounded, $\left(b_{n}^{\prime \prime \prime}\right)_{n \in \mathbb{N}}$ is bounded, too. By [3, page 110, Theorem 2.4], for $x_{0} \notin(d, e)$, we have

$$
0<\frac{Q_{n+1}\left(x_{0}\right)}{\left(x_{0}-b_{n}^{\prime}-b^{\prime}\right) Q_{n}\left(x_{0}\right)} \leq 1, \quad n \geq 0 .
$$

Hence, $\left|a_{n}^{\prime \prime \prime}\right|<\left|\left(x_{0}-b_{n}^{\prime}-b^{\prime}\right) / Q_{1}\left(x_{0}\right)\right|$, which shows the boundedness of $\left(a_{n}^{\prime \prime \prime}\right)$. Finally, $a_{n}^{\prime \prime \prime}+b_{n}^{\prime \prime \prime}+c_{n}^{\prime \prime \prime}=1$ yields the boundedness of $\left(c_{n}^{\prime \prime \prime}\right)$. By Theorem 6.2 (iii), the proof is complete.

Now, let us examine the duals of an SBG polynomial hypergroup. We define the sets

$$
\begin{aligned}
& D_{r}=\left\{z \in \mathbb{C},\left|P_{n}(z)\right| \leq r \gamma(n) \text { for all } n \in \mathbb{N}_{0}\right\}, \quad D=\bigcup_{r \geq 1} D_{r}, \\
& D_{r}^{s}=D_{r} \cap \mathbb{R}, \quad \text { and } \quad D^{s}=\bigcup_{r \geq 1} D_{r}^{s} .
\end{aligned}
$$

Furthermore we define for some $z \in \mathbb{C}$ the function $\alpha_{z}(n)=P_{n}(z)$ for all $n \in \mathbb{N}_{0}$. Then the following theorem holds.

THEOREM 6.5. Let $\mathcal{K}=\left\{P_{n}, n \in \mathbb{N}_{0}\right\}$ be an $S B G$ polynomial hypergroup.

(i) It holds that $\mathcal{X}^{b}\left(\mathbb{N}_{0}\right)=\left\{\alpha_{z}, z \in D\right\}$ and $\widehat{\mathbb{N}}_{0}=\left\{\alpha_{x}, x \in D^{s}\right\}$.

(ii) The mappings

$$
D \rightarrow \mathcal{X}^{b}\left(\mathbb{N}_{0}\right), \quad z \mapsto \alpha_{z} \quad \text { and } \quad D^{s} \rightarrow \widehat{\mathbb{N}}_{0}, \quad x \mapsto \alpha_{x}
$$

are homeomorphisms. 
(iii) $\mathcal{X}^{h}\left(\mathbb{N}_{0}\right)$ and $\widehat{\mathbb{N}}_{0}$ are bounded.

PROOF. (i) For $z \in D_{r}$ it holds that $\left\|\alpha_{z}\right\|_{\infty, \gamma} \leq r, \alpha_{z} \neq 0$, see [3, Chapter I, Theorem 5.3], and $L_{n} \alpha_{z}(m)=\alpha_{z}(n) \alpha_{z}(m)$, hence $\left\{\alpha_{z}, z \in D_{r}\right\} \subset \mathcal{X}_{r}^{b}\left(\mathbb{N}_{0}\right)$.

Now suppose $\alpha \in \mathcal{X}_{r}^{b}\left(\mathbb{N}_{0}\right)$ and put $z=a_{0} \alpha(1)+b_{0}$. We obtain $\alpha(1) \alpha(n)=$ $L_{1} \alpha(n)=a_{n} \alpha(n+1)+b_{n} \alpha(n)+c_{n} \alpha(n-1)$. Since $\alpha(0)=1$ and $\alpha(1)=\left(z-b_{0}\right) / a_{0}$, $\alpha(n)$ satisfies the same recurrence relation as $P_{n}(z)$, hence they must be equal. This yields $\mathcal{X}_{r}^{b}\left(\mathbb{N}_{0}\right) \subset\left\{\alpha_{z}, z \in D_{r}\right\}$. Note that $P_{n}(z)=\overline{P_{n}(z)}$ for all $n \in \mathbb{N}_{0}$ implies $z \in \mathbb{R}$.

(ii) Let $V\left(\alpha_{z_{0}}, \epsilon, n_{1}, \ldots, n_{k}\right)=\left\{\alpha \in \mathcal{X}^{b}(K),\left|\alpha\left(n_{i}\right)-\alpha_{z_{0}}\left(n_{i}\right)\right|<\epsilon, i=1, \ldots, k\right\}$. Clearly, its inverse under the mapping $z \mapsto \alpha_{z}$ is the set

$$
\bigcap_{i=1}^{k}\left\{z \in D,\left|P_{n_{i}}(z)-P_{n_{i}}\left(z_{0}\right)\right|<\epsilon\right\},
$$

which is open. Since $\mathcal{X}^{h}\left(\mathbb{N}_{0}\right)$ is equipped with the topology of pointwise convergence the mapping $\mathcal{X}^{b}\left(\mathbb{N}_{0}\right) \rightarrow D, \alpha_{z} \mapsto a_{0} \alpha_{z}(1)+b_{0}$ is continuous, too. The second statement follows since $\widehat{\mathbb{N}}_{0}$ bears the induced topology.

(iii) Let $B$ denote the bound of $\left(\left|a_{n}\right|\right),\left(\left|b_{n}\right|\right)$ and $\left(\left|c_{n}\right|\right)$, and choose $M>0$ such that the zeros $z_{n, 1}, z_{n, 2}, \ldots, z_{n, n}$ of any $P_{n}$ are elements of the interval $[-M, M]$. We have $P_{n}(z)=\alpha_{n} \prod_{i=1}^{n}\left(z-z_{n . i}\right)$ with $\alpha_{n}=\left(a^{n} \prod_{i=1}^{n-1} a_{i}\right)^{-1}$. Choose $z \in D$ and assume $|z|>M$. Then there exists $r \geq 1$ such that $\left|\alpha_{n}\right| \prod_{i=1}^{n}\left|z-z_{n, i}\right| \leq r \gamma(n)$. Since $\left|z-z_{n, i}\right| \geq|z|-M$ we get $(|z|-M)^{n} \leq r \gamma(n) / \alpha_{n}$. By Lemma 6.1 , we are able to deduce $\gamma(n)=\mathcal{O}\left(n(|a| B)^{n}\left|\alpha_{n}\right|\right)$. Therefore there exists $C>0$ such that $|z|-M \leq|a| B \sqrt[n]{r C n}$ for all $n \in \mathbb{N}$, which implies $|z| \leq M+|a| B$.

We would like to mention the question of whether the dual of an SBG polynomial hypergroup that is compact is still open.

\section{Jacobi polynomials}

The Jacobi polynomials $P_{n}^{(\alpha, \beta)}$ are orthogonal with respect to the measure

$$
\pi(x)=(1-x)^{\alpha}(1+x)^{\beta} d x, \text { for all } \alpha, \beta>-1,
$$

with $\operatorname{supp} \pi=[-1,1]=\mathcal{S}$. According to Theorem 6.4 they form a normalized SBG polynomial hypergroup when normalizing at a point $x_{0} \notin(-1,1)$.

In case $x_{0}=1$, the three term recurrence relation coefficients are given by

$$
a=\frac{2(\alpha+1)}{\alpha+\beta+2}, \quad b=\frac{\beta-\alpha}{\alpha+\beta+2},
$$




$$
\begin{aligned}
& a_{n}=\frac{(n+\alpha+\beta+1)(n+\alpha+1)(\alpha+\beta+2)}{(2 n+\alpha+\beta+2)(2 n+\alpha+\beta+1)(\alpha+1)}, \\
& b_{n}=\frac{\alpha-\beta}{2(\alpha+1)}\left[1-\frac{(\alpha+\beta+2)(\alpha+\beta)}{(2 n+\alpha+\beta+2)(2 n+\alpha+\beta)}\right], \\
& c_{n}=\frac{n(n+\beta)(\alpha+\beta+2)}{(2 n+\alpha+\beta+1)(2 n+\alpha+\beta)(\alpha+1)},
\end{aligned}
$$

see [8]. The corresponding normalized polynomials are denoted by $R_{n}^{(\alpha, \beta)}$ and we compute

$$
\begin{aligned}
& h(0)=1, \\
& h(n)=\frac{(2 n+\alpha+\beta+1) \Gamma(\alpha+\beta+n+1) \Gamma(\alpha+n+1) \Gamma(\beta+n+1)}{(n+1) \Gamma(\alpha+\beta+2) \Gamma(\alpha+1) \Gamma(\beta+1)}, \quad n \in \mathbb{N} .
\end{aligned}
$$

By using Stirling's formula we get

$$
h(n)=\mathcal{O}\left(n^{2 \alpha+1}\right)
$$

If $(\alpha, \beta) \in V=\{(\alpha, \beta), \alpha \geq \beta>-1, \alpha \geq-1 / 2\}$ then $\gamma$ is bounded, see [1, Theorem 1], that is, $\left(R_{n}^{(\alpha, \beta)}\right)_{n \in \mathbb{N}_{0}}$ constitutes a discrete signed hypergroup. Furthermore, if

$$
\begin{aligned}
(\alpha, \beta) \in W= & \left\{(\alpha, \beta), \alpha \geq \beta, a(a+5)(a+3)^{2} \geq\left(a^{2}-7 a-24\right) b^{2}\right\} \\
& \supset\{(\alpha, \beta), \alpha \geq \beta>-1, \alpha+\beta+1 \geq 0\},
\end{aligned}
$$

where $a=\alpha+\beta+1$ and $b=\alpha-\beta$, then $\left(R_{n}^{(\alpha, \beta)}\right)_{n \in \mathbb{N}_{0}}$ constitutes a discrete hypergroup, see [6, Theorem 1].

By switching the normalization point $x_{0}$ to -1 and denoting the corresponding polynomials by $S_{n}^{(\alpha, \beta)}$, we have $S_{n}^{(\alpha, \beta)}(x)=R_{n}^{(\beta, \alpha)}(-x)$, see also [6, page 585]. Hence, when $(\beta, \alpha) \in V$, then $S_{n}^{(\alpha, \beta)}$ constitute a discrete signed hypergroup and when $(\beta, \alpha) \in W$ they form a discrete hypergroup.

The remaining region is $G=\{(\alpha, \beta),-1<\alpha, \beta<-1 / 2\}$. Making use of Theorem 6.4, (7.1) and $\gamma(n) \geq \max \left(h(n)^{-1}, 1\right)$ we deduce for $(\alpha, \beta) \in G$ that both $\left\{R_{n}^{(\alpha, \beta)}, n \in \mathbb{N}_{0}\right\}$ and $\left\{S_{n}^{(\alpha, \beta)}, n \in \mathbb{N}_{0}\right\}$ form an SBG polynomial hypergroup which is not bounded.

For the ultraspherical polynomials, that is, $\alpha=\beta$, we will determine $\gamma(n)$ explicitly for $-1<\alpha<-1 / 2$.

THEOREM 7.1. Let $-1<\alpha<-1 / 2$. For $\gamma$ corresponding with $R_{n}^{(\alpha, \alpha)}$ it holds that $\gamma(0)=\gamma(1)=1$ and for $n \geq 2$

$$
\gamma(n)=\sum_{k}|g(n, n, k)|=\frac{2}{\prod_{k=1}^{n-1} a_{k}}\left(\prod_{k=n}^{2 n-1} a_{k}+\prod_{k=1}^{n} c_{k}\right)-1<4 h(n)^{-1}-1 .
$$


In particular, there exist constants $C_{1}, C_{2}>0$ such that

$$
C_{1} n^{-2 \alpha-1} \leq \gamma(n) \leq C_{2} n^{-2 \alpha-1} .
$$

ProOF. We make use of Theorem 6.4, (7.1) and $\gamma(n) \geq \max \left(h(n)^{-1}, 1\right)$ to show the correspondence with an SBG hypergroup which is not bounded.

It is clear that $\gamma(0)=\gamma(1)=1$. We use Lemma 6.1 to deduce for $n \geq m \geq 2$ that $g(n, m, n-m+2 j-1)=0, j=1,2, \ldots, m, g(n, m, n-m), g(n, m, n-m)>0$ and $g(n, m, n-m+2 j)<0, j=1,2, \ldots, m-1$. Hence, for all $n, m \geq 2$,

$$
\sum_{k}|g(n, m, k)|=2(g(n, m,|n-m|)+g(n, m, n+m))-1 .
$$

Let $2 \leq m<n$. Using (6.3) and (6.4) we derive

$$
\sum_{k} g|(n, m, k)|<\sum_{k} g|(n, m+1, k)| .
$$

Now suppose $2 \leq n \leq m$ and set $r_{m}=\prod_{k=m}^{m+n-1} a_{k}+\prod_{k=m-n+1}^{m} c_{k}$. Since

$$
\sum_{k}|g(n, m, k)|=2 r_{m}\left(\prod_{k=1}^{n-1} a_{k}\right)^{-1}-1,
$$

the inequality $r_{m+1}<r_{m}$ yields $\sum_{k}|g(n, m+1, k)|<\sum_{k}|g(n, m, k)|$. Finally, we derive

$$
\gamma(n)=\sum_{k}|g(n, n, k)|=\frac{2}{\prod_{k=1}^{n-1} a_{k}}\left(\prod_{k=n}^{2 n-1} a_{k}+\prod_{k=1}^{n} c_{k}\right)-1 \text { for all } n \geq 2 .
$$

By using $\prod_{k=n}^{2 n-1} a_{k}<\prod_{k=1}^{n} c_{k}$ this yields $h(n)^{-1} \leq \gamma(n)<4 h(n)^{-1}-1$. With (7.1) we get the last assertion.

Now it is straightforward to determine the dual objects of the generalized hypergroups generated by ultraspherical polynomials.

THEOREM 7.2. Let $-1<\alpha$. Then the duals of the generalized hypergroup $\left\{R_{n}^{(\alpha, \alpha)}\right.$, $\left.n \in \mathbb{N}_{0}\right\}$ of ultraspherical polynomials coincide, $\mathcal{S}=\widehat{\mathbb{N}}_{0}=\mathcal{X}^{b}\left(\mathbb{N}_{0}\right) \simeq[-1,1]$.

Proof. We have to show $\mathcal{S} \supset \mathcal{X}^{b}(K)$. Assume $z \in \mathbb{C} \backslash[-1,1]$. From [15, Equation (8.21.9)], we deduce that $R_{n}(z)$ grows exponentially with $n$. From (7.2) we know that $\gamma(n)$ grows only polynomially. Hence, there does not exist a constant $r$ such that $\left|R_{n}(z)\right| \leq r \gamma(n)$, which means $\alpha_{z} \notin \mathcal{X}^{b}\left(\mathbb{N}_{0}\right)$. 
One might ask the question what happens in the case $(\alpha, \beta) \in G$ when choosing the normalization point $c \notin[-1,1]$. Surprisingly, $[1$, Theorem 2$]$ immediately yields the following theorem.

THEOREM 7.3. Let $(\alpha, \beta) \in G$ and choose $c \in \mathbb{B} \backslash[-1,1]$. The Jacobi polynomials $T_{n}^{(\alpha, \beta)}$ normalized at $c\left(T_{n}^{(\alpha, \beta)}(c)=1\right)$ constitute a normalized and bounded generalized hypergroup, that is, a discrete signed hypergroup. The duals are given by

$$
\begin{aligned}
\mathcal{S} & =[-1,1], \quad \widehat{\mathbb{N}}_{0}=[-|c|,|c|], \\
\mathcal{X}^{b}\left(\mathbb{N}_{0}\right) & =\left\{z \in \mathbb{C},\left|z+\sqrt{z^{2}-1}\right| \leq|c|+\sqrt{c^{2}-1}\right\} .
\end{aligned}
$$

Hereby, the branch of $\sqrt{z^{2}-1}$ is chosen such that $\left|z+\sqrt{z^{2}-1}\right| \geq 1$.

\section{Acknowledgement}

We thank Ryszard Szwarc for fruitful discussions with respect to the boundedness properties of the dual of an SBG polynomial hypergroup.

\section{References}

[1] R. Askey and G. Gasper, 'Linearization of the product of Jacobi polynomials III', Canad. J. Math. 23 (1971), 332-338.

[2] W. R. Bloom and H. Heyer, Harmonic analysis of probability measures on hypergroup (de Gruyter, Berlin, 1995).

[3] T. S. Chihara, An introduction to orthogonal polynomials (Gordon and Breach, New York, 1978).

[4] C. Dunkl, 'The measure algebra of a locally compact hypergroup', Trans. Amer. Math. Soc. 179 (1973), 331-348.

[5] F. Filbir, R. Lasser and J. Obermaier, 'Summation kernels for orthogonal polynomials', in: Handbook on analytic-computational methods in applied mathematics (ed. G. Anastassiou) (Chapman and Hall, Boca Raton, 2000) pp. 709-749.

[6] G. Gasper, 'Linearization of the product of Jacobi polynomials Il', Canad. J. Math. 22 (1970), 582-593.

[7] R. I. Jewett, 'Spaces with an abstract convolution of measures'. Adv. Math. 18 (1975), 1-101.

[8] R. Lasser, 'Orthogonal polynomials and hypergroups'. Rend. Mat. (7) 3 (1983), 185-209.

[9] R. Lasser and M. Leitner, 'Stochastic processes indexed by hypergroups I', J. Theoret. Probab. 2 (1989), 301-311.

[10] R. Lasser and J. Obermaier, 'On the convergence of weighted Fourier expansions', Acta. Sci. Math. (Szeged) 61 (1995), 345-355.

[11] M. Leitner, 'Stochastic processes indexed by hypergroups II', J. Theoret. Probab. 4 (1991), 321331.

[12] N. Obata and N. J. Wildberger, 'Generalized hypergroups and orthogonal polynomials', Nagoya Math. J. 142 (1996), 67-93. 
[13] K. A. Ross, 'Signed hypergroups - a survey', Contemp. Math. 183 (1995), 319-329.

[14] R. Spector, Apercu de la Théorie des Hypergroupes, Lecture Notes in Math.. Vol. 497 (Analyse Harmonique sur les Groupes de Lie, Sem. Nancy-Strasbourg 1973-1975) (Springer, Berlin, 1975).

[15] G. Szegö, Orthogonal polynomials (Amer. Math. Soc., New York, 1959).

[16] D. Werner, Funktionalanalysis (Springer, Berlin, 1995).

GSF-National Research Center

for Environment and Health

Institute of Biomathematics and Biometry

Ingolstädter Landstrasse 1

D-85764 Neuherberg

Germany

e-mail: lasser@gsf.de josef.obermaier@gsf.de
University of Vienna Faculty of Mathematics

NuHAG

Nordbergstr. 15

A-1090 Vienna

Austria

e-mail: holger.rauhut@univie.ac.at 
